\title{
SASKATCHEWAN CHRISTMAS MAMMAL COUNT — 1983
}

Compiled by WAYNE C. HARRIS, Box 414, Raymore, Saskatchewan. SOA 3J0

A total of 55 counts were received this year reporting 32 species during the count period. The number of counts exceeds the previous high of 49 in 1982 and the number of species exceeds the high of 28 recorded in 1978, 1980 and 1982. These new high totals are in spite of the particularly harsh weather during this count period.

Although no new species were added to the Christmas mammal count list there were four species seen only once in the past ten years. These were the Fisher at Prince Albert National Park, Fox Squirrel at Regina, Gray Squirrels at Saskatoon and Northern Flying Squirrel at Good Spirit Lake.

Populations are similar to last year. Lynx were not reported. Only Whitetailed Jack Rabbit and Snowshoe Hare show declines over last year. The apparent decline in Red Squirrels may be due to the extremely cold weather rather than a real decrease in population.

The only species for which a noticeable increase occurred is Nuttall's Cottontail, for which more than usual were seen and over a greater range. Is it a coincidence that cottontail numbers seemingly are increasing while hare population decline? Do cottontail populations peak when hares are at their low?

In spite of the cold weather during much of the count period both Striped Skunks and Badgers were active. Is this normal, regardless of weather?

For weather, coverage and partici- pants on these counts please refer to the Christmas Bird Counts found elsewhere in this issue. Symbols found in the table are as follows:

* Identified by tracks with estimated number of individuals in parentheses

+ Seen during count period but not on count day

L Active lodges or huts seen with number in parentheses

$\mathrm{H}$ Species heard but not seen with number in parentheses

$S$ Presence indicated by odor with number in parentheses

D Fresh diggings seen

d Freshly dead animal seen

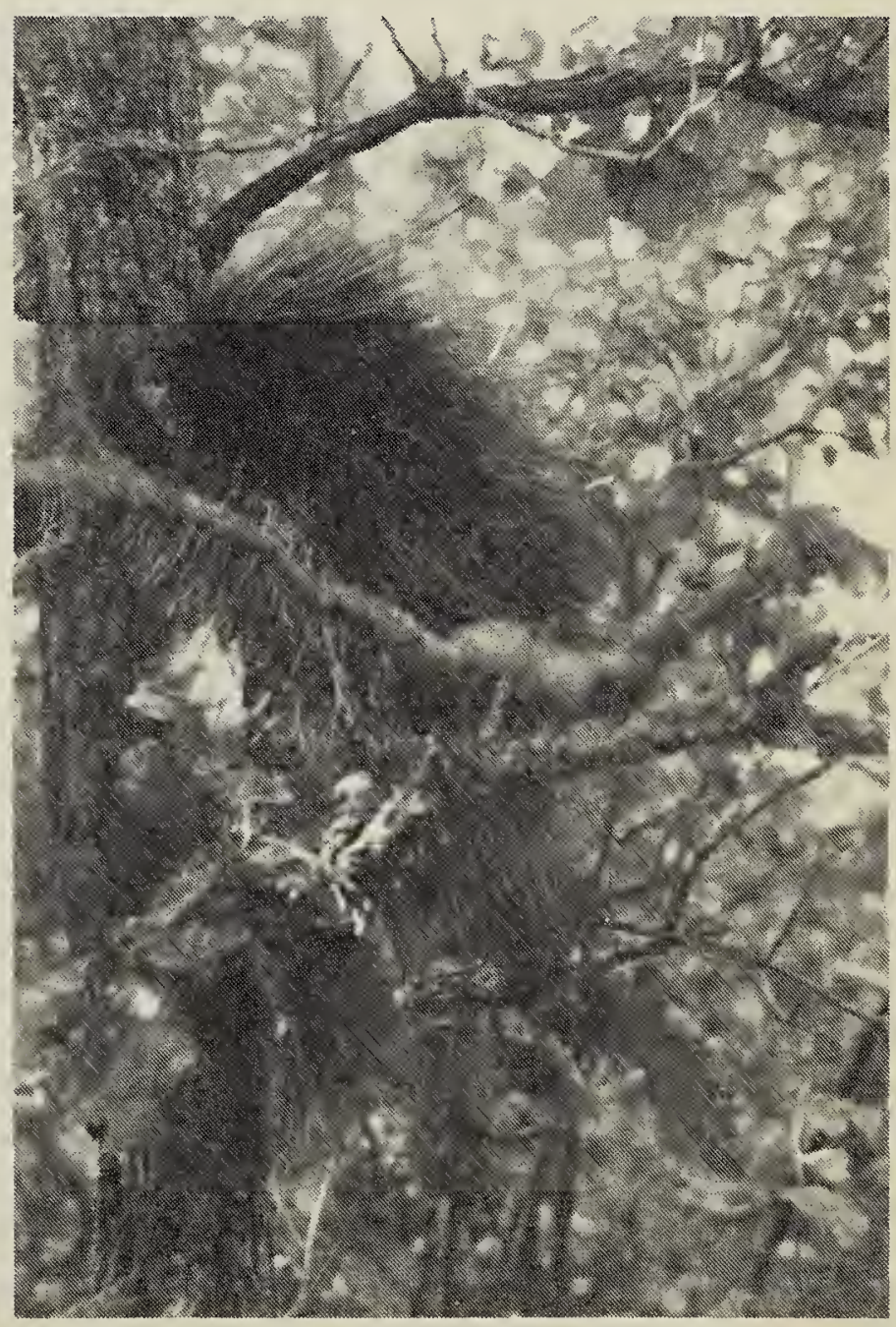

Porcupine

G.J. Smith 
Table 1-1. SASKATCHEWAN CHRISTMAS MAMMAL COUNTS

\section{SPECIES}

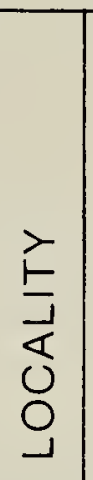

SHREW species

RACOON

RED FOX

COYOTE

TIMBER WOLF

FISHER

ERMINE

LEAST WEASEL

LONG-TAILED WEASEL

WEASEL species

MINK

RIVER OTTER

STRIPED SKUNK

BADGER

WHITE-TAILED JACKRABBIT SNOWSHOE HARE

NUTTALL'S COTTONTAIL

FOX SQUIRREL

RED SQUIRREL

GRAY SQUIRREL

N. FLYING SQUIRREL

BEAVER

DEER MOUSE

MEADOW VOLE

MOUSE species

MUSKRAT

NORWAY RAT

HOUSE MOUSE

PORCUPINE

ELK

MULE DEER

WHITE-TAILED DEER

DEER species

MOOSE

PRONGHORN

TOTAL SPECIES

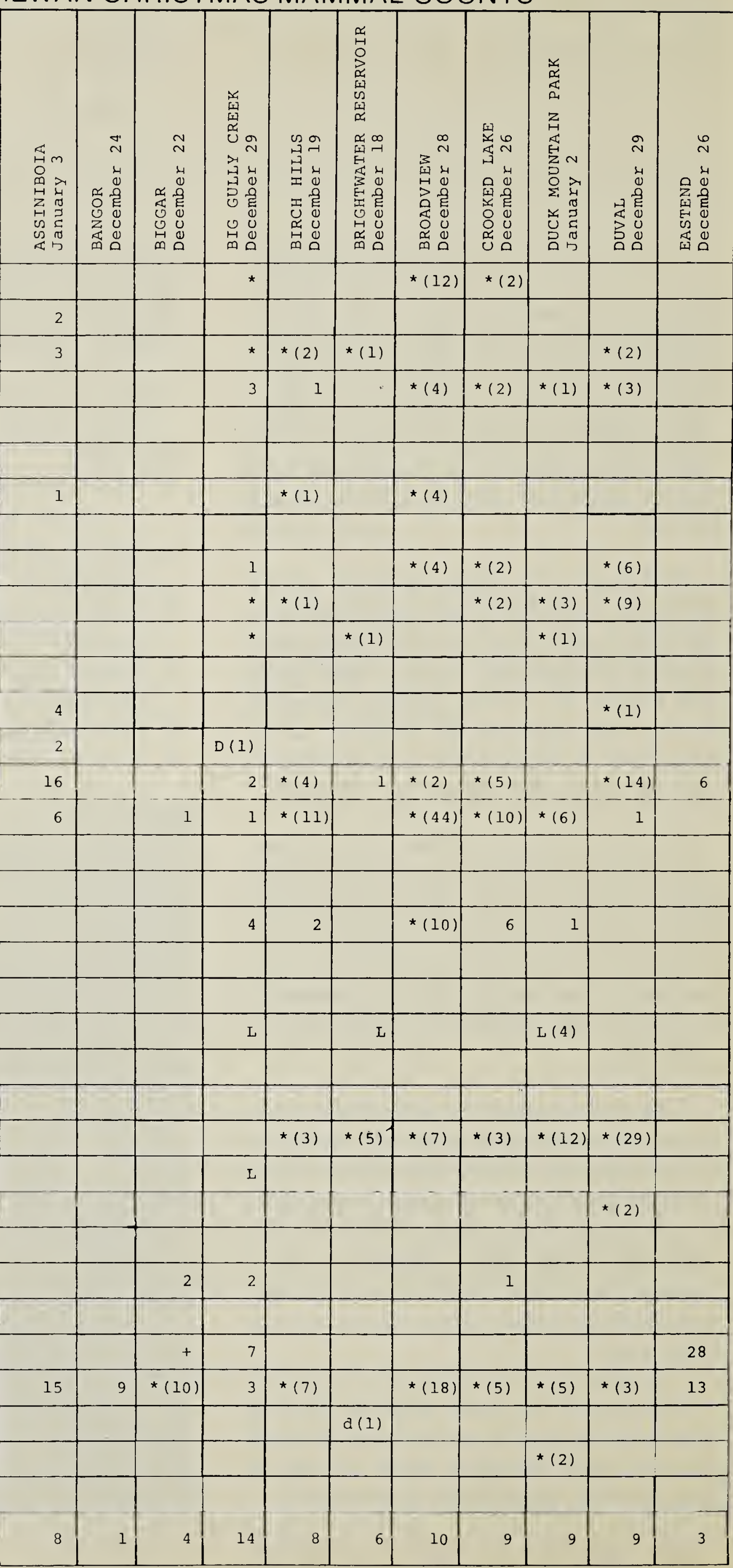


Table 1-2. SASKATCHEWAN CHRISTMAS MAMMAL COUNTS

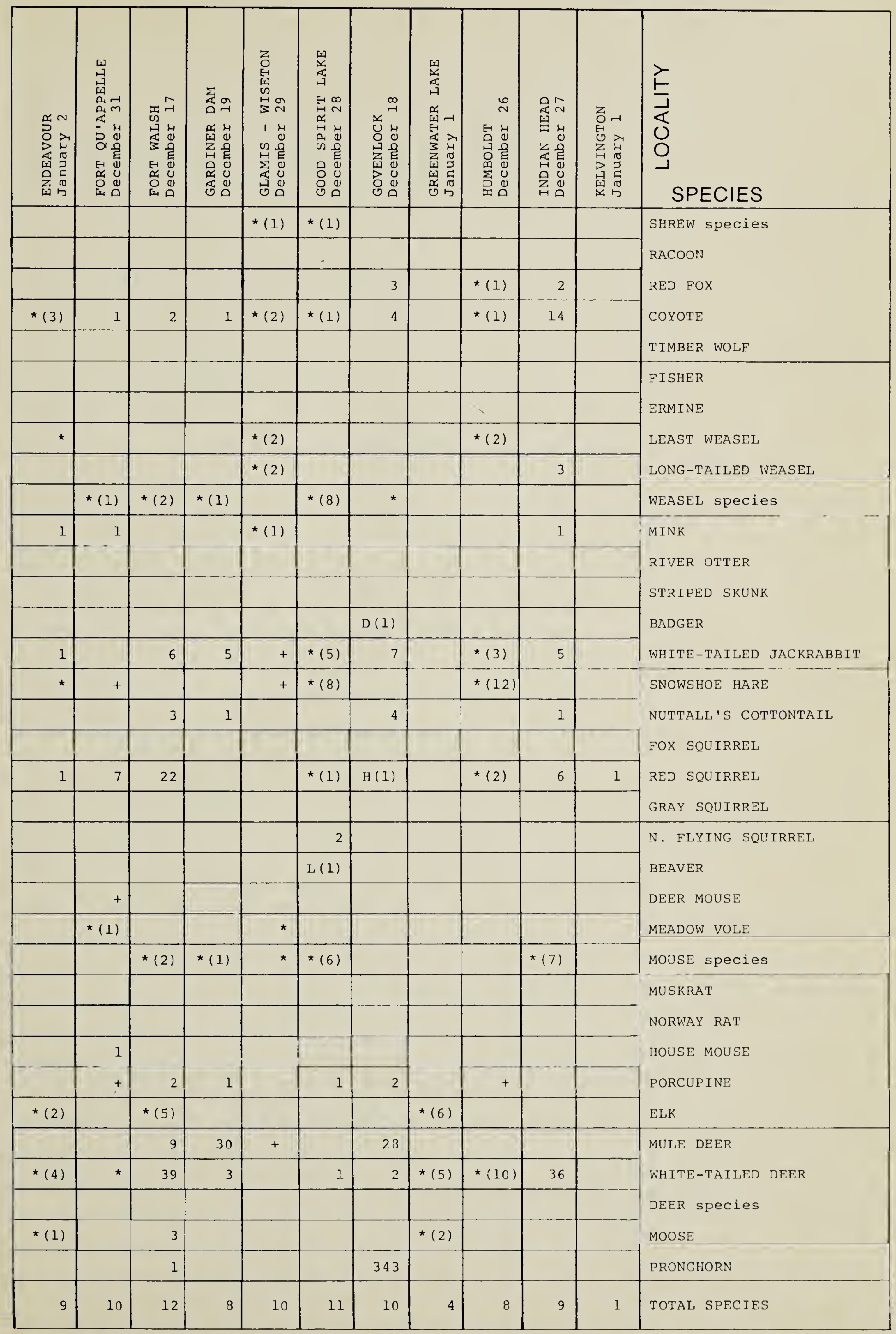


Table 1-3. SASKATCHEWAN CHRISTMAS MAMMAL COUNTS

\section{SPECIES}

SHREW species

RACOON

RED FOX

COYOTE

TIMBER WOLF

FISHER

ERMINE

LEAST WEASEL

LONG-TAILED WEASEL

WEASEL species

MINK

RIVER OTTER

STRIPED SKUNK

BADGER

WHITE-TAILED JACKRABBIT

SNOWSHOE HARE

NUTTALL'S COTTONTAIL

FOX SQUIRREL

RED SQUIRREL

GRAY SQUIRREL

N. FLYING SQUIRREL

BEAVER

DEER MOUSE

MEADOW VOLE

MOUSE species

MUSKRAT

NORWAY RAT

HOUSE MOUSE

PORCUPINE

ELK

MULE DEER

WHITE-TAILED DEER

DEER species

MOOSE

PRONGHORN

TOTAL SPECIES

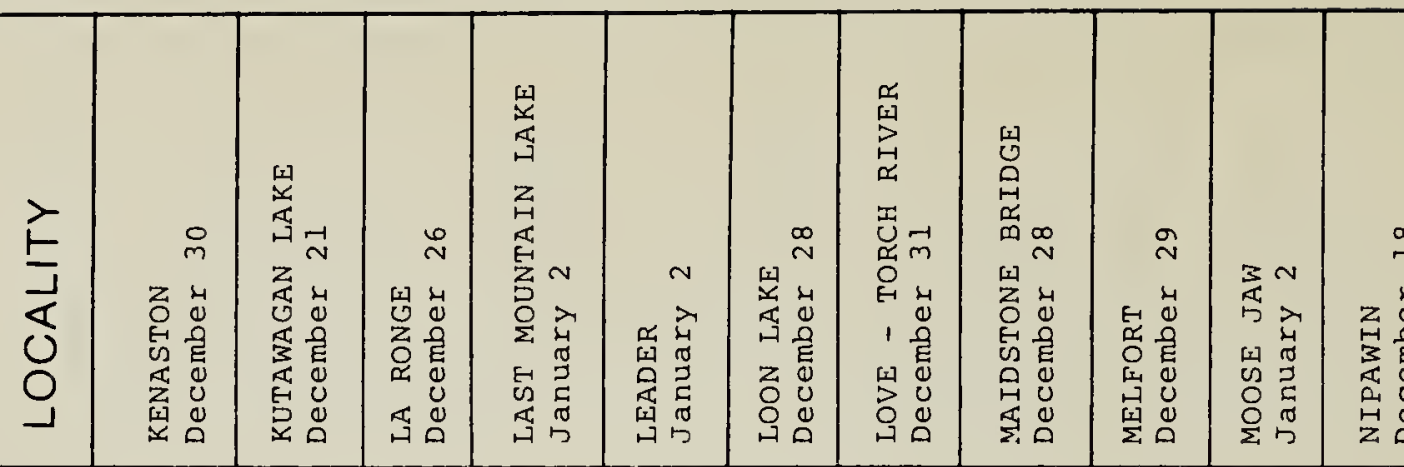

$\stackrel{\infty}{-1}$

\begin{tabular}{|l|r|r|r|r|r|r|r|r|r|r|}
\hline & & & & & & & & & & \\
\hline$*(15)$ & 1 & $*(1)$ & $*$ & & & $*(1)$ & $*$ & & 3 & \\
\hline$*(1)$ & 2 & & 2 & 2 & 1 & $*(10)$ & 2 & & 3 & $*(5)$ \\
\hline
\end{tabular}

\begin{tabular}{l|l|l|l|l|l}
\hline & & & & & 2 \\
\hline & & & & & \\
\hline
\end{tabular}

*(1)

*(1)

*(2)

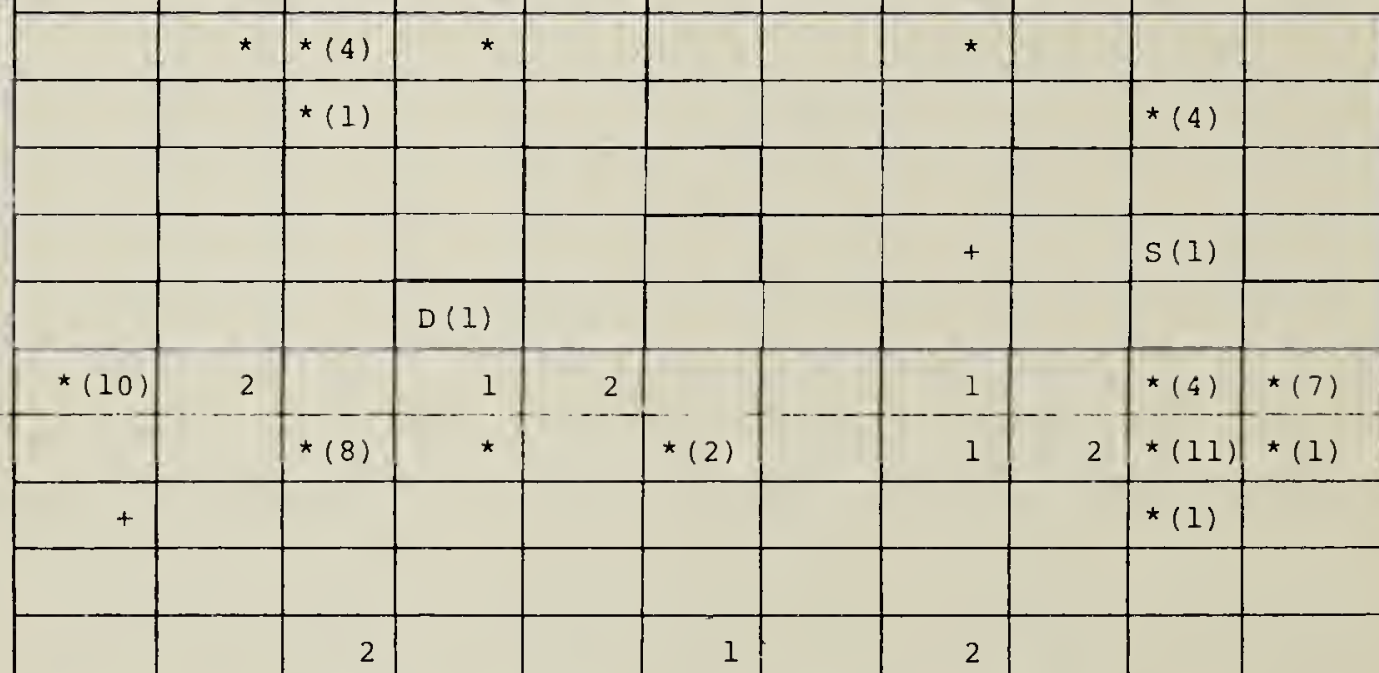


Table 1-4. SASKATCHEWAN CHRISTMAS MAMMAL COUNTS

\begin{tabular}{|c|c|c|c|c|c|c|c|c|c|c|c|}
\hline 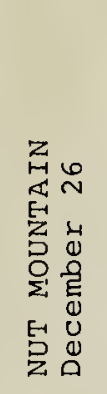 & 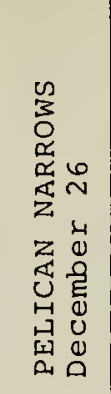 & 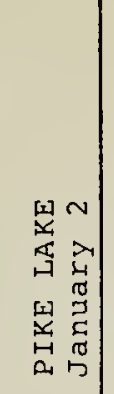 & 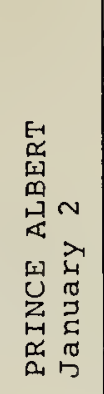 & 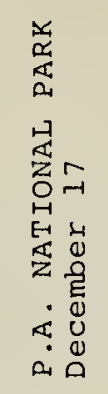 & 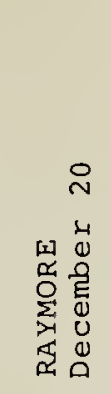 & 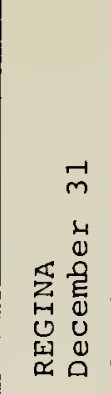 & 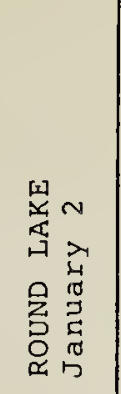 & 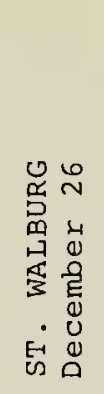 & 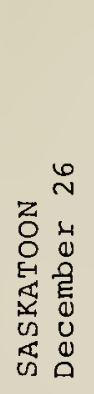 & 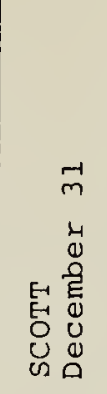 & 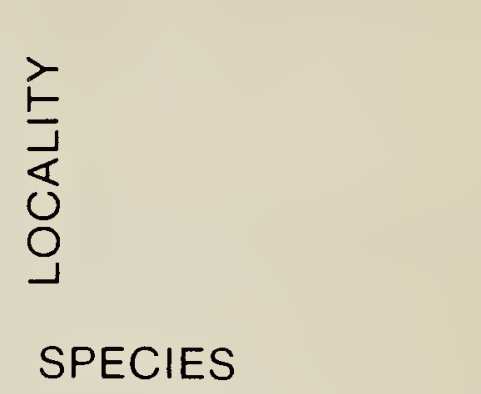 \\
\hline & & & & & $\star$ & $\star(1)$ & & & & & \multirow{5}{*}{$\begin{array}{l}\text { SHREW species } \\
\text { RACOON } \\
\text { RED FOX } \\
\text { COYOTE } \\
\text { TIMBER WOLF }\end{array}$} \\
\hline & & & & & & & & & & & \\
\hline$\star(3)$ & + & & 2 & 5 & $\star$ & 1 & * $(6)$ & 1 & & & \\
\hline \multirow[t]{5}{*}{ * $(1)$} & & $\mathrm{H}(2)$ & $\mathrm{H}$ & & 2 & & $*(12)$ & 2 & 2 & 1 & \\
\hline & + & & & 2 & & & & & & & \\
\hline & & & & $\star(1)$ & & & & & & & \multirow{4}{*}{$\begin{array}{l}\text { FISHER } \\
\text { ERMINE } \\
\text { LEAST WEASEL } \\
\text { LONG-TAILED WEASEL }\end{array}$} \\
\hline & & & & & & $*(3)$ & $*(12)$ & & & & \\
\hline & & & & & & $*(1)$ & $\star(5)$ & & & & \\
\hline \multirow[t]{6}{*}{$\star(1)$} & & & & & 1 & $*(7)$ & $\star(18)$ & 1 & & & \\
\hline & $\star(1)$ & $*(2)$ & & & 1 & & & & 1 & & \multirow{5}{*}{$\begin{array}{l}\text { WEASEL species } \\
\text { MINK } \\
\text { RIVER OTTER } \\
\text { STRIPED SKUNK } \\
\text { BADGER } \\
\text { WHITE-TAILED JACKRABBIT }\end{array}$} \\
\hline & + & & & $\star(1)$ & * & & $*(6)$ & & & & \\
\hline & & & & & + & & & & & & \\
\hline & & & & & + & & & & & & \\
\hline & & $\star(1)$ & & & 6 & 14 & $*(1)$ & & 4 & 1 & \\
\hline \multirow[t]{6}{*}{ *(2) } & $*(3)$ & 4 & & $*(3)$ & 3 & & 2 & $\star(5)$ & 3 & & \multirow{5}{*}{$\begin{array}{l}\text { SNOWSHOE HARE } \\
\text { NUTTALL'S COTTONTAIL } \\
\text { FUX SQUIRKEL } \\
\text { RED SQUIRREL } \\
\text { GRAY SQUIRREL }\end{array}$} \\
\hline & & & & & & & & & & & \\
\hline & & & & & & + & & & & & \\
\hline & 2 & 1 & & 2 & 2 & & 3 & & & & \\
\hline & & & & & & & & & 2 & & \\
\hline & & & & & & & & & & & \multirow{4}{*}{$\begin{array}{l}\text { N. FLYING SQUIRREL } \\
\text { BEAVER } \\
\text { DEER MOUSE } \\
\text { MEADOW VOLE }\end{array}$} \\
\hline \multirow[t]{4}{*}{$L(1)$} & & & & & $L(1)$ & & & & & & \\
\hline & & & & & 2 & & & & & & \\
\hline & + & & & & 1 & * & & & & & \\
\hline & + & * (19) & & *(1) & & * & * & & * & $\star(2)$ & MOUSE species \\
\hline \multirow[t]{6}{*}{$L(6)$} & $\star(I)$ & & & & $L(7)$ & & & & 1 & & \multirow{4}{*}{$\begin{array}{l}\text { MUSKRAT } \\
\text { NORWAY RAT } \\
\text { HOUSE MOUSE } \\
\text { PORCUPINE }\end{array}$} \\
\hline & & & & & $\star$ & & & & & & \\
\hline & & & & & 1 & & & & & & \\
\hline & & 1 & & & 3 & $\star(1)$ & 4 & & 1 & & \\
\hline & & & & 33 & & & & & & & ELK \\
\hline & & $\star(5)$ & & & & & & & & & \multirow{5}{*}{$\begin{array}{l}\text { MULE DEER } \\
\text { WHITE-TAILED DEER } \\
\text { DEER species }\end{array}$} \\
\hline+ & & & & 1 & 5 & & $\star(12)$ & * $(3)$ & 2 & & \\
\hline & & $\star(5)$ & & & & & & & & & \\
\hline & & & & $\star(1)$ & & & & $\star(2)$ & & & \\
\hline & & & & & & & & & & & \\
\hline 7 & 8 & 8 & 2 & 10 & 18 & 8 & 12 & 6 & 9 & 3 & TOTAL SPECIES \\
\hline
\end{tabular}


Table 1-5. SASKATCHEWAN CHRISTMAS MAMMAL COUNTS

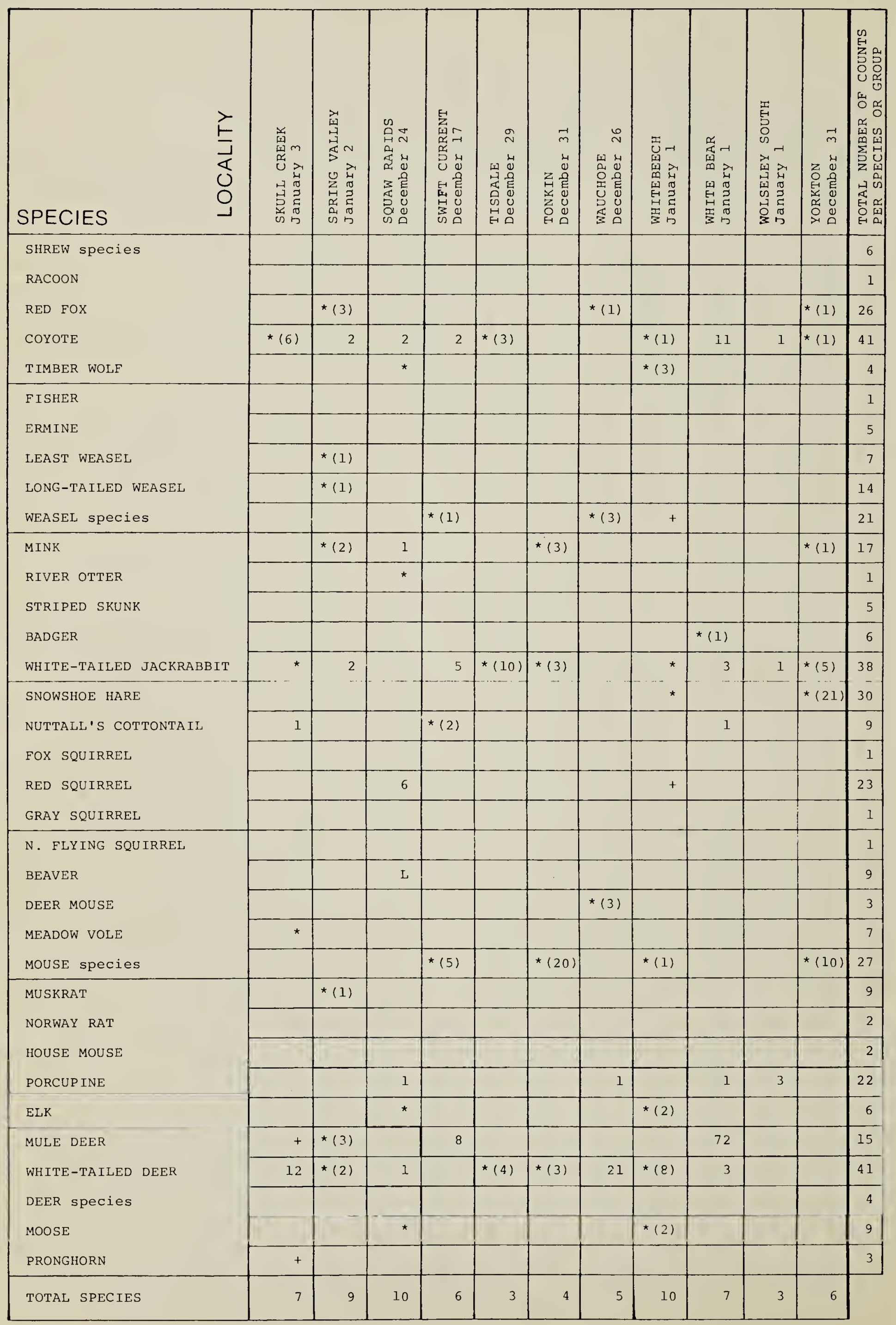

